\title{
A rare case of untreated tubercular spondylodisctis
}

\section{Colangelo*, G Autore, V Pambianco, VM Formica, LA Nasto, and E Pola}

Catholic University of Rome, Italy

A 44 years-old Romanian man presented at our Emergency Department with productive cough, fever with chills, profuse sweating, and chronic dorsal pain. He referred previous history of tuberculosis with progressive dorsal gibbus deformity treated only with antibiotics several years before in his country. The patient underwent blood tests and chest X-ray that confirmed the suspect of recurrent pulmonary tuberculosis. The physical examination assessed no neurologic deficits. After being isolated, the patient underwent full spine MRI that showed the consequences of previous Pott's disease extended from T8 to T12 vertebral levels. As shown in the figure, D11 vertebral body was

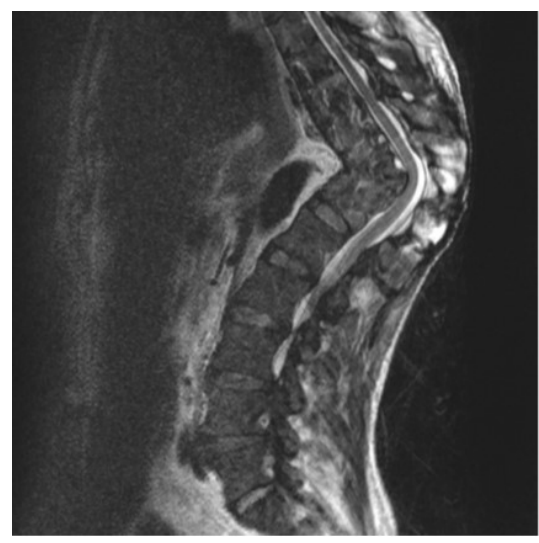

Copyright: (C)2016 Colangelo D. This is an open-access article distributed under the terms of the Creative Commons Attribution License, which permits unrestricted use, distribution, and reproduction in any medium, provided the original author and source are credited. completely collapsed causing a segmental kyphosis with a Cobb Angle of $82,12^{\circ}$ and posterior spinal cord tenting with no signal changes. A TC-guided biopsy confirmed the microbiological diagnosis. The patient was treated for the recurrent pulmonary tuberculosis with a combination of antibiotic drugs with complete healing after 40 days of therapy.

The MRI findings show the potential severe deformity associated with Pott disease. The risks associated with a surgical correction of the segmental kyphosis were unacceptable due to the absence of neurological impairment and the relative stability of his deformity.

The severity of dorsal hyperkyphosis shown in figure is a very rare occurrence in industrialized countries and this radiological finding shows the potential consequences of untreated Pott disease.
Correspondence to: Debora Colangelo, Orthopaedic Surgeon, Catholic University of Rome, Italy, Tel: +39- 320-46-95-741, E-mail: debora.colangelo@gmail.com

Received: November 01, 2016; Accepted: November 17, 2016; Published: November 19, 2016 